\title{
Validación de la Escala de Fortalezas Familiares en población adulta chilena
}

\author{
Joucelyn Rivadeneira-Valenzuela; Marcelo M. Rivadeneira
}

How to cite this article:

Rivadeneira-Valenzuela, J., \& Rivadeneira, M. M. (2021). Validación de la Escala de Fortalezas Familiares en población adulta chilena. Acta Colombiana de Psicología, 24(2), 120-129. https://www.doi.org/10.14718/ACP.2021.24.2.11

Recibido, enero 07/2020; Concepto de evaluación, abril 07/2021; Aceptado mayo 20/2021

\author{
Joucelyn Rivadeneira-Valenzuela ${ }^{1}$ \\ ORCID: http://orcid.org/0000-0001-6917-1690 \\ Universidad Arturo Prat, Iquique, Chile. \\ Marcelo M. Rivadeneira \\ ORCID: https://orcid.org/0000-0002-1681-416X \\ Centro de Estudios Avanzados en Zonas Áridas (CEAZA), Coquimbo, Chile. \\ Universidad Católica del Norte, Coquimbo, Chile. \\ Universidad de La Serena, La Serena, Chile.
}

\begin{abstract}
Resumen
El enfoque de las fortalezas familiares sugiere que las familias son capaces de surgir y salir exitosas de los desafíos de la vida cotidiana. Teniendo esto en cuenta, el objetivo de este estudio fue validar la Escala de Fortalezas Familiares, adaptada de un estudio en España, en una población chilena. Para esto, la muestra estuvo formada por 324 adultos chilenos que debían completar el instrumento de 12 ítems. El modelo de dos factores de la escala fue validado mediante un análisis factorial confirmatorio $\left(\chi^{2} / \mathrm{gl}=2.86\right.$; RMSR $=0.041 ;$ RMRSEA $\left.=0.076 ; \mathrm{CFI}=0.950 ; \mathrm{TLI}=0.937\right)$, y se encontró que los dos factores se relacionan con aspectos de la autoeficacia familiar y la percepción de adaptación en las familias; en ambos casos se estimaron valores de confiabilidad de escala adecuados (alfa de Cronbach de .91 y .72, respectivamente). Estos resultados permiten avalar la utilización de la escala en la población chilena, lo que podrá contribuir en el avance del conocimiento respecto al funcionamiento familiar, así como guiar intervenciones de prevención y promoción con familias dirigidas a potenciar fortalezas y recursos protectores garantes de una parentalidad respetuosa y afectiva, y al mejoramiento de la calidad de vida familiar.

Palabras clave: fortalezas familiares, funcionamiento familiar, autoeficacia, adaptación, recursos familiares.
\end{abstract}

\section{Validation of the Family Strengths Scale in Chilean adult population}

\begin{abstract}
The family strength approach suggests that families can overcome daily life challenges. The aim of this research was to validate the Family Strengths Scale, adapted from a study in Spain, to a Chilean population. For this purpose, the sample consisted of 324 Chilean adults who completed a 12-item questionnaire. The two-factor model of the scale was validated by confirmatory factor analysis $\left(\chi^{2} / \mathrm{g} .1 .=2.86\right.$; RMSR $=0.041$; RMREA $=0.076$; CFI $=0.950$; TLI $\left.=0.937\right)$, and it was found that the two factors are related to aspects of family self-efficacy and the perception of family adaptation. In both cases, adequate scale reliability values were estimated (Cronbanch's alpha of 0.91 and 0.72 , respectively). These results support the use of the scale in the Chilean population, which may contribute to the advancement of knowledge regarding family functioning, as well as to guide prevention and promotion interventions with families, aimed at enhancing strengths and protective resources that guarantee a respectful and affectionate parenting, and at improving the quality of family life. Keywords: family strengths, family functioning, self-efficacy, adaptation, family resources.
\end{abstract}

1 Facultad de Ciencias Jurídicas y Políticas, Universidad Arturo Prat, Iquique, Chile. Avenida Arturo Prat 2120, Iquique, Chile. Tel.: (56)5726697.jrivadenei@unap.cl 


\section{Introducción}

El enfoque de las fortalezas familiares (en adelante $\mathrm{FF}$ ) se construye sobre la base de que todas las familias poseen las capacidades, habilidades y fortalezas para construir en conjunto un sistema que crece, cambia y se desarrolla, y consiste en un enfoque que fomenta la salud y el bienestar en general, incluida la resiliencia entre los individuos y las familias (Fruhauf et al., 2020). En este sentido, las FF se plantean como un claro cambio de paradigma que apuesta por dejar atrás el concepto de familia disfuncional y el enfoque centrado en el déficit o daño que esta posee (Bagur \& Verger, 2020; Yeung \& Chan, 2011); de manera que esta nueva perspectiva respecto a la familia encuentra sustento en la psicología positiva, pues cuenta con una mirada centrada en las cualidades, fortalezas y el bienestar psicosocial del ser humano (Baker \& van Woerkom, 2018; Sheridan et al., 2017).

En particular, las FF se han entendido como las competencias y capacidades individuales y grupales que presenta la familia como respuesta frente a situaciones de crisis y estrés, así como para promover y aumentar las fortalezas del funcionamiento del sistema familiar (Ghaffari, 2016; Yeung et al., 2012). No obstante, mediante el enfoque de las FF no se pretende minimizar las dificultades que existen en la familia, sino que se trata de abordar el funcionamiento de esta desde una perspectiva más optimista y equilibrada del mundo, y procura comprender cómo es que las familias enfrentan los desafíos (Julien-Chinn et al., 2017).

Existen diversos modelos que evalúan las características que suelen estar presentes en las familias con mayores fortalezas, pero en general se ha encontrado que son familias que poseen un clima de comunicación positiva, que presentan alta capacidad para la resolución de conflictos, así como un sentido espiritual con profundas creencias y valores que les permiten afrontar de manera exitosa el estrés, y que cuentan con un fuerte compromiso con el desarrollo de la familia a través de sus miembros (Davenport et al., 2014; Olson \& Olson, 2000; Schumm et al., 2001). Asimismo, otros modelos han explorado dimensiones en estas familias que se relacionan con factores protectores y estresores que estas vivencian, entre ellos las demandas, la valoración, el apoyo, el afrontamiento y la adaptación (Drummond et al., 2002; Gardner et al., 2008). Desde estas dimensiones, se ha encontrado que una familia que posee alta tolerancia a los factores estresores tendrá una visión más adaptativa, lo que le permite a sus miembros desarrollar una mejor autoestima, optimismo, habilidades y creatividad (Gardner et al., 2008). Teniendo esto en cuenta, vemos que este enfoque puede ser de mucha utilidad, especialmente en población vulnerable (Samson, 2018), dado que los enfoques tradicionales suelen centrarse en las deficiencias del individuo y la familia, lo que da como resultado una mayor desmoralización y estigmatización.

Ahora bien, de forma mayoritaria, las investigaciones en el ámbito de las FF se han desarrollado en contextos europeos y en Estados Unidos. Como consecuencia de ello, existen grandes avances en materia de promoción de programas de intervención profesional que buscan abordar el trabajo con familias de esos contextos, con énfasis en mejorar las habilidades sociales, emocionales y de bienestar de sus miembros, y que puedan a su vez ayudar a mejorar la calidad de vida familiar (Churchill \& Kieckhefer, 2018; Kumpfer et al., 2018; Valdez et al., 2013), muy especialmente en grupos de alto riesgo social (Kumpfer \& Magalhaes, 2018). De igual forma, se ha valorado el impacto positivo de dichos programas en la salud mental y física de los hijos, pues se han reportado menores niveles de estrés parental y depresión a partir de dichas intervenciones (Fitzgerald et al., 2011; Kieckhefer et al., 2014; McKelvey et al., 2015; Valdez et al., 2013).

Adicionalmente, el funcionamiento familiar se refiere a las propiedades estructurales y sociales del ambiente global de la familia, incluidas las interacciones de sus miembros, sus conflictos, la cohesión, la adaptabilidad, la organización y su comunicación (McKelvey et al., 2015). En este sentido, la evaluación del funcionamiento familiar ha permitido una mejor comprensión de la diversidad y complejidad de las interacciones sociales que se dan en el espacio familiar, $\mathrm{y}$ ha sido de especial utilidad para los equipos de trabajo que desarrollan sus tareas en contextos sociales complejos (Julien-Chinn et al., 2017; Kumpfer \& Magalhaes, 2018; Roque \& Acle, 2012), en especial cuando se trata del desarrollo de los hijos (Souza \& Aparecida, 2019).

Al respecto, la evaluación de las FF se ha utilizado, en conjunto con la resiliencia familiar, como elemento que facilita la comprensión del funcionamiento familiar (McKelvey et al., 2015; Sánchez et al., 2019; Valdez et al., 2013; Walsh, 2016). De hecho, la resiliencia familiar se entiende como la capacidad de los sistemas familiares de emplear sus fortalezas colectivas para resistir, crecer y recuperarse de las adversidades (Masten, 2018), y su análisis surgió en contextos vulnerables en los cuales los niños y sus familias no presentaban problemas a pesar de las difíciles condiciones en las que vivían (Sánchez et al., 2019); no obstante, la investigación al respecto se ha ampliado al conocimiento de diversas áreas que involucran la salud y el trabajo de las personas en general (Soler et al., 2016; Walsh, 2016). 
Pese a la importancia de las FF para abordar el trabajo con familias, la evidencia en la población nacional es escasa, aunque se puede destacar que en Chile el estudio en torno a la familia ha ido en aumento en las últimas décadas, con especial énfasis en la creación y adaptación de instrumentos evaluativos (Bello-Escamilla et al., 2017; Cumsille et al., 2014; Gubbins \& Ibarra, 2016; Rivadeneira-Valenzuela et al., 2021; Rivadeneira \& López, 2017). Incluso, respecto a la evaluación del funcionamiento familiar, también existen importantes avances en el ámbito nacional que permiten acercar de cierta forma la brecha empírica que nos separa del resto de investigaciones internacionales en esta materia, ya que se encuentran estudios que abordan aspectos de la resiliencia familiar (Peña \& Molina, 2013), su relación con el ámbito de la salud en general (Salas et al., 2010; Vicente et al., 2012), de la salud mental (De la Barra et al., 2012; Valdivia et al., 2015), y la relación entre familia y trabajo (Figueroa et al., 2011; Riquelme et al., 2012).

Sin perjuicio de lo anterior, a la fecha no se conocen investigaciones que involucren las FF, ni se cuenta con un instrumento estandarizado que evalúe dichas características para la población chilena. Por esto, el presente estudio tiene como propósito validar la Escala de Fortalezas Familiares - elaborada originalmente por Olson et al. (1982) y validada en España por Sanz et al. (2002) - en Chile, con el fin de hacer frente al vacío empírico existente en el país.

\section{Método}

\section{Tipo de estudio y diseño}

La presente investigación se adscribe a un estudio de tipo instrumental. Al respecto, Montero y León (2005) señalan que este tipo de estudios son los encargados del "desarrollo de pruebas y aparatos, incluyendo tanto el diseño o adaptación como el estudio de las propiedades psicométricas de los mismos" (p. 124).

\section{Participantes}

El estudio tuvo como base el registro total de estudiantes escolarizados entre los niveles educativos de cuarto a sexto año de educación básica de la ciudad de Chillán, en Chile, durante el año 2015, que en ese momento ascendía a 5124. La ciudad cuenta con 33 establecimientos educativos municipales y 35 establecimientos educativos en modalidad subvencionada-particular. La muestra del estudio se obtuvo a partir de una selección aleatoria simple de tres establecimientos educacionales por unidad administrativa. Se realizó un muestreo por conglomerados, teniendo en cuenta los niveles cuarto, quinto y sexto básico, con lo cual la muestra quedó compuesta por 324 madres, padres o adultos responsables de los jóvenes de esos niveles. Por otro lado, los criterios de inclusión del estudio se centraron en las familias con hijos o hijas pertenecientes a niveles cuarto, quinto y sexto de educación básica que accedieron voluntariamente al estudio, previa firma del consentimiento informado.

\section{Procedimiento}

Tanto las familias como los directores de los establecimientos educativos recibieron información sobre los objetivos del estudio; además, se les solicitó la firma del consentimiento informado a los padres, madres y adultos para participar voluntariamente en el mismo. Previo a la aplicación del instrumento, los encuestadores — estudiantes de último año de Licenciatura en Trabajo Social- se sometieron a entrenamiento supervisado por parte de los investigadores con el objetivo de uniformar el método de recogida de los datos, en el que se incluyó la forma de administrar el instrumento. La aplicación se llevó a cabo de forma colectiva en las reuniones de cada nivel, programadas por los establecimientos educativos.

\section{Aspectos éticos}

Este estudio fue revisado y aprobado por el Comité de Ética de la Universidad del Bío-Bío, para ajustarse a los estándares de estudios con personas en Chile.

\section{Instrumentos}

La Escala de Fortalezas Familiares evalúa los recursos con los que cuenta una familia. Es original de Olson et al. (1982) y fue adaptada a España por Sanz et al. (2002) bajo el nombre de Escala de Recursos Familiares. Su objetivo es obtener una medida de las fortalezas y capacidades que los miembros de una familia tienen para hacer frente a diferentes estresores. En este sentido, los recursos son considerados características del sistema familiar, incluidos aspectos sociológicos, emocionales y físicos que hacen que la familia sea menos vulnerable y, por lo tanto, más capaz de afrontar los estresores y de adaptarse, sea de manera individual o familiar. Desde un punto de vista teórico, esta escala puede tener capacidad predictiva debido a que un mayor grado de recursos familiares puede predecir un mejor funcionamiento familiar.

En su versión española, la escala presenta un $\alpha$ de Cronbach de .85 para la escala total y de .84 y .55 para las subescalas, así como valores test-retest de .87 y .54 para las subescalas y de .91 para la escala total. En particular, en la versión española, la subescala I está conformada por los ítems 1, 2, 3, 4, 5, 7, 8, 10, 12 (véase Tabla 1), y la subescala 
II está conformada por los ítems 6, 9 y 11 (no se encuentra una interpretación de los mismos por parte de los autores).

Esta escala ha sido utilizada a nivel internacional en diversos estudios (Greeff, 2000; Sanz, 2003; Thompson \& Schrodt, 2015), y consta de 12 ítems (véase Tabla 1) con respuesta tipo Likert, donde cada ítem se puntúa sobre una escala con cinco alternativas de respuesta: 1 = "no describe nada a mi familia", 2 = "solo la describe ligeramente", 3 = "describe a veces a mi familia", 4 = "en general, sí describe a mi familia", y 5 = "describe muy bien a mi familia". A mayor puntaje, mayor nivel de recursos y fortalezas familiares.

Adicional a esta escala, se aplicó un breve cuestionario generado especialmente para el presente estudio para obtener los datos sociodemográficos de la muestra.

\section{Análisis estadísticos}

La matriz de datos original fue filtrada para dejar solo cuestionarios completos ( $n=324$ casos). Según la propuesta de Wolf et al. (2013), este tamaño de muestra es adecuado para satisfacer una solución factorial confirmatoria de dos factores, con un poder de 1- $\beta>0.86$. Para evaluar la fiabilidad de la escala se empleó el índice $\alpha$ de Cronbach (Hair et al., 2014), y para evaluar la factibilidad de llevar a cabo el análisis factorial se estimó el factor de adecuación Kaiser-MeyerOlkin (KMO) y la prueba de esfericidad de Bartlett — valores de KMO $>0.8$ son considerados aceptables (Kaiser \& Rice, 1974), y se espera que la prueba de Bartlett arroje valores de probabilidad $<0.05$ - ; además, se realizó un análisis factorial confirmatorio (AFC), cuya validez fue evaluada mediante los siguientes indicadores: razón $\chi^{2}$-grados de libertad $(\mathrm{gl}<3)$ - preferible al uso del valor de probabilidad de $\chi^{2}$, dada su dependencia a tamaños de muestra elevados-(Alavi et al., 2020), la raíz del residuo cuadrático medio (RMSR <.08), la raíz del error cuadrático medio de aproximación (RMRSEA $<$ .08 ), el índice de ajuste comparativo (CFI $>0.95$ ) y el índice de Tucker-Lewis (TLI > 0.95) (Kline, 2016). Los análisis fueron realizados empleando las librerías Pysch, versión 1.8.4 (Revelle, 2018), y lavaan (Rossel, 2012) en R 3.5 (R Core Team, 2018).

Tabla 1.

Valor de correlaciones (producto-momento de Spearman) entre items

\begin{tabular}{|c|c|c|c|c|c|c|c|c|c|c|c|c|}
\hline Ítems & RF_1 & RF_2 & RF 3 & RF_4 & RF_5 & RF_6 & RF_7 & RF_8 & RF_9 & RF_10 & RF_11 & RF_12 \\
\hline $\begin{array}{l}\mathrm{RF} \_1 \text {. Podemos expresar nues- } \\
\text { tros sentimientos. }\end{array}$ & 1.00 & & & & & & & & & & & \\
\hline $\begin{array}{l}\text { RF_2. Nos sentimos capaces } \\
\text { de afrontar problemas cuando } \\
\text { surgen. }\end{array}$ & .57 & 1.00 & & & & & & & & & & \\
\hline $\begin{array}{l}\text { RF_3. Tenemos confianza en } \\
\text { nosotros mismos. }\end{array}$ & .61 & .60 & 1.00 & & & & & & & & & \\
\hline $\begin{array}{l}\text { RF_4. Tenemos confianza en } \\
\text { que podemos resolver los pro- } \\
\text { blemas eficazmente. }\end{array}$ & .55 & .62 & .70 & 1.00 & & & & & & & & \\
\hline $\begin{array}{l}\text { RF_5. Los miembros de mi } \\
\text { familia somos leales a ella. }\end{array}$ & .56 & .54 & .61 & .58 & 1.00 & & & & & & & \\
\hline $\begin{array}{l}\text { RF_6. Conseguir nuestros } \\
\text { objetivos nos resulta fácil. }\end{array}$ & .50 & .39 & .48 & .53 & .42 & 1.00 & & & & & & \\
\hline $\begin{array}{l}\text { RF 7. Los miembros de mi fa- } \\
\text { milia decimos lo que pensamos } \\
\text { de los otros de forma positiva. }\end{array}$ & .41 & .34 & .50 & .48 & .42 & .46 & 1.00 & & & & & \\
\hline $\begin{array}{l}\mathrm{RF} \text { R. Compartimos valores y } \\
\text { creencias muy similares. }\end{array}$ & .46 & .49 & .60 & .58 & .64 & .44 & .48 & 1.00 & & & & \\
\hline $\begin{array}{l}\text { RF_9. Creemos que las cosas } \\
\text { nos van bien en nuestra familia. }\end{array}$ & .39 & .36 & .40 & .49 & .36 & .42 & .37 & .38 & 1.00 & & & \\
\hline $\begin{array}{l}\text { RF_10. Los miembros de mi } \\
\text { familia nos respetamos entre } \\
\text { nosotros. }\end{array}$ & .52 & .47 & .58 & .53 & .56 & .47 & .45 & .55 & .49 & 1.00 & & \\
\hline $\begin{array}{l}\text { RF_11. Pocos problemas quedan } \\
\text { por resolver en nuestra familia. }\end{array}$ & .48 & .42 & .47 & .50 & .37 & .52 & .42 & .35 & .45 & .48 & 1.00 & \\
\hline $\begin{array}{l}\text { RF_12. Nos sentimos orgullosos } \\
\text { de nuestra familia. }\end{array}$ & .43 & .45 & .51 & .48 & .62 & .38 & .41 & .47 & .40 & .54 & .41 & 1.00 \\
\hline
\end{tabular}

Nota. Todas las correlaciones fueron significativas $(p<.001)$. 


\section{Resultados}

Los principales datos descriptivos de la población indican que la edad promedio de los adultos fue de 40.14 años $(D T=10.18)$. Del total de participantes, el $83.8 \%$ era madre, el $13.8 \%$, padre, y el $2.4 \%$, otro adulto responsable. Además, el $19.1 \%$ reportó tener estudios básicos, el $50.9 \%$, estudios de enseñanza media, y el $28.2 \%$, nivel de estudios universitarios y posuniversitarios. Además, respecto a la situación laboral, el $55.1 \%$ se encontraba laboralmente activo. Otros resultados de este estudio han sido publicados anteriormente (Rivadeneira \& López, 2017).

En general, la matriz de datos contiene 12 ítems, para un total de 324 observaciones, lo que indica una razón de 27 observaciones por variable. Todas las correlaciones entre los 12 ítems fueron positivas $(M=.48$; rango $=.34-.70)$ y significativas a un valor de probabilidad menor a .001 (véase Tabla 1).

En la Tabla 2 se presentan las puntuaciones medias y la variabilidad de cada ítem que integra la escala aplicada a la muestra de adultos chilenos. Las correlaciones entre el puntaje de cada ítem con el total de la escala variaron entre .56 a .76, donde los ítems RF_7 y RF_9 tuvieron correlaciones $<.60$. Además, los valores $\alpha$ de Cronbach de la escala exhiben valores altos (entre .90 y .91), incluso al eliminar los ítems con menor correlación. Por otra parte, el coeficiente de adecuación muestral (MSA) presentó valores entre .91 y .96, el índice de adecuación muestral KMO tuvo un valor de 0.94, y la prueba de esfericidad de Barlett fue significativa $\left(\chi^{2}=1984.96, \mathrm{gl}=66, p<.0001\right)$.

Finalmente, el modelo de dos factores fue validado mediante un AFC, tras el cual se incluyó, además, el ajuste de un modelo unifactorial para efectos comparativos (véase Tabla 3). El AFC de dos factores exhibió valores de $\alpha$ de Cronbach de .91 (autoeficacia) y .72 (percepción de adaptación), tras lo cual se observó que el modelo presentó un buen ajuste para cuatro de los cinco índices de ajuste $\left(\chi^{2} / \mathrm{gl}=2.86\right.$, RMSR $=0.041$, RMRSA $=0.076$, CFI $\left.=0.95\right)$. Solo el índice de Tucker-Lewis tuvo un tuvo un valor inferior $(\mathrm{TLI}=0.937)$, aunque aun cercano al valor umbral $(\mathrm{TLI}=0.95)$; en contraste, el modelo unifactorial exhibió valores de $\chi^{2} / \mathrm{gl}>3$, RMRSEA $>0.08$, y CFI y TLI $<0.95$. Como se puede observar en la Figura 1, las cargas del modelo bifactorial se muestran empleando un modelo de ecuaciones estructurales.

\section{Discusión}

Respecto a estos hallazgos, el instrumento que se presenta permite distinguir dos factores o dimensiones que componen las FF para la muestra evaluada - la autoeficacia y la percepción de adaptación -, que han sido anteriormente descritas en la literatura en torno a la evaluación de las FF (Drummond et al., 2002; Gardner et al., 2008; McKelvey

Tabla 2.

Medidas de resumen, correlación item total (r), alfa de Cronbach si se elimina el ítem, y coeficiente de adecuación muestral (MSA) de todos los items de la escala

\begin{tabular}{cccccc}
\hline Ítem & Media & $D E$ & $r$ & Alfa & MSA \\
\hline RF_1 & 4.44 & 0.81 & .69 & .90 & 0.94 \\
RF_2 & 4.49 & 0.82 & .65 & .91 & 0.94 \\
RF_3 & 4.55 & 0.74 & .76 & .90 & 0.94 \\
RF_4 & 4.48 & 0.75 & .76 & .90 & 0.93 \\
RF_5 & 4.69 & 0.63 & .70 & .90 & 0.91 \\
RF_6 & 3.74 & 0.88 & .63 & .91 & 0.95 \\
RF_7 & 4.22 & 0.81 & .59 & .91 & 0.96 \\
RF_8 & 4.50 & 0.77 & .67 & .90 & 0.94 \\
RF_9 & 4.18 & 0.99 & .56 & .91 & 0.94 \\
RF_10 & 4.53 & 0.78 & .70 & .90 & 0.95 \\
RF_11 & 4.03 & 0.98 & .61 & .91 & 0.94 \\
RF_12 & 4.79 & 0.56 & .63 & .91 & 0.93 \\
\hline
\end{tabular}

Tabla 3.

Índices de bondad de ajuste para dos modelos de análisis factorial confirmatorio evaluados, considerando uno y dos factores

\begin{tabular}{cccccccccc}
\hline Modelo & $\chi^{2}$ & $\mathrm{gl}$ & $\chi^{2} / \mathrm{gl}$ & Valor de $p$ & RMSR & RMRSEA & CFI & TLI \\
\hline Un factor & 177.69 & 54 & 3.29 & $<.001$ & 0.045 & 0.084 & 0.937 & 0.923 \\
Dos factores & 151.69 & 53 & 2.86 & $<.001$ & 0.041 & 0.076 & 0.950 & 0.937 \\
\hline
\end{tabular}


Figura 1.

Análisis factorial confirmatorio basado en un modelo de dos factores

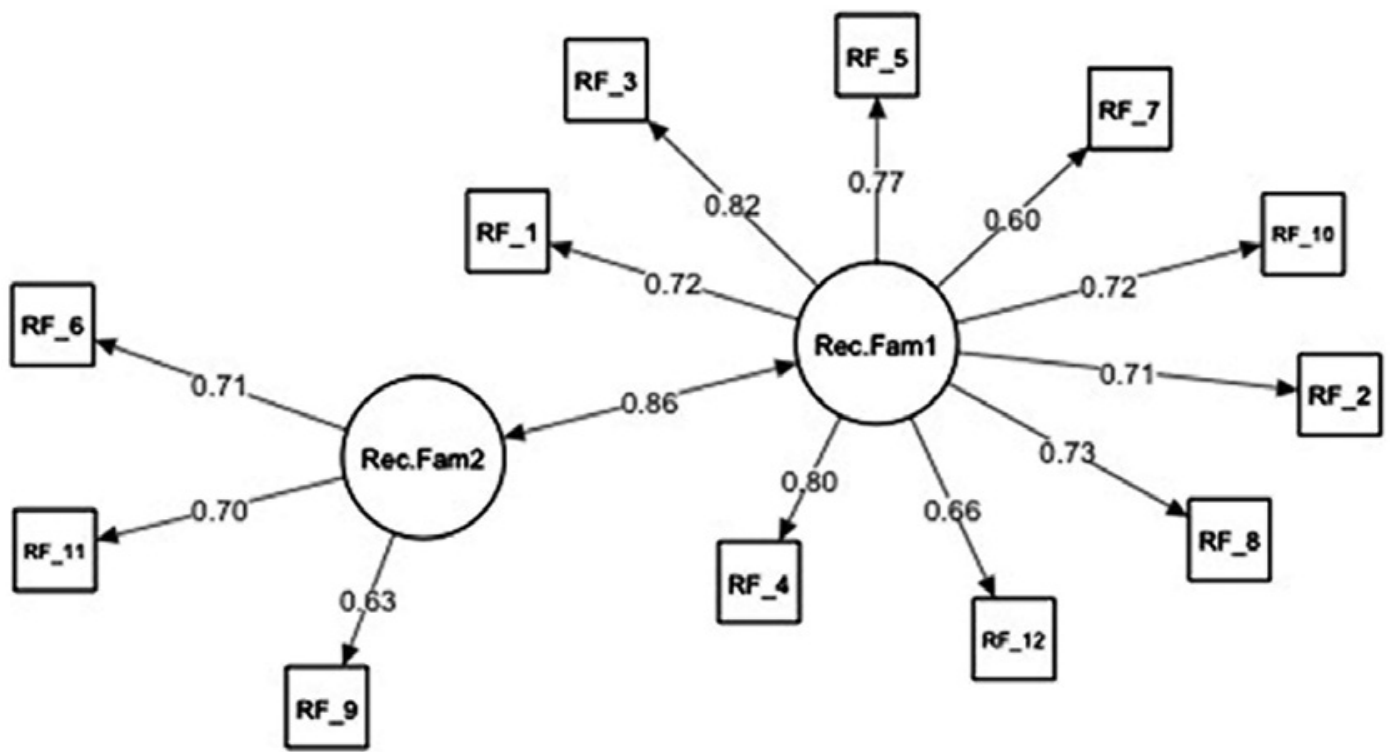

Nota. Se indican las cargas de los ítems asociados a cada factor (flechas unidireccionales) y la correlación entre factores (flecha bidireccional).

et al., 2015; Olson \& Olson; 2000; Schumm et al., 2001). Ambas dimensiones encontradas coinciden, además, con el instrumento en su versión española, aunque en dicho estudio no se presentó una posible interpretación de ambas dimensiones.

Específicamente, la autoeficacia refiere a la percepción que tienen los miembros de la familia respecto a la confianza en las propias competencias que les permiten salir adelante frente a elementos estresores (Bandura, 2000). Al respecto, la investigación en torno a la autoeficacia familiar muestra que los padres y madres con mayor grado de percepción de autoeficacia responden de mejor manera a la conducta de los hijos, a la vez que proporcionan mayor apoyo afectivo, comunicativo y material, y con ello crean las condiciones familiares necesarias para favorecer su desarrollo integral (McKelvey et al., 2015; Spielmann \& Taubman-Ben-Ari, 2009; Vega \& de Dios, 2006). Asimismo, una mayor percepción de autoeficacia parental presenta implicaciones positivas en la calidad de vida y la salud mental familiar (Gilliam \& Steffen, 2006). Adicional a esto, la autoeficacia y el dominio de los padres para actuar se han asociado con relaciones entre padres e hijos más óptimas y una crianza más respetuosa (Sanders \& Woolley, 2005).

En tanto a la percepción de adaptación, esta incluye las expectativas positivas que la familia posee, así como la sensación de calma y manejo que permite aceptar y lidiar con las situaciones cotidianas (Drummond et al., 2002; Gardner et al., 2008; McKelvey et al., 2015). En este sentido, la adaptación familiar es el resultado del desarrollo continuo que se produce entre procesos protectores familiares exitosos y procesos de vulnerabilidad en la vida familiar (Drummond et al., 2002). Si entendemos la familia como una unidad multidimensional compleja y en constante cambio, podemos reconocer que su desarrollo y funcionamiento dependen en gran medida de la capacidad que posean los miembros de dicha familia para proporcionarse cuidado mutuo, vínculos afectivos para manejar situaciones de estrés, y propender a la adaptación durante los tiempos de adversidad.

El hallazgo de una alta correlación entre ambas dimensiones puede encontrar respuesta en estudios anteriores, donde dichas variables han sido reconocidas como parte de las características de lo que se consideran las FF, ya que ambas suelen ser incluidas en los programas preventivos con base en el modelo de las fortalezas (Kumpfer et al., 2018; Kumpfer \& Magalhaes, 2018; McKelvey et al., 2015). En efecto, en dichos programas de fortalecimiento familiar se refuerza la empatía, la comunicación efectiva, la organización de los miembros de la familia, las habilidades para manejar los conflictos, y, en el fondo, promover una mejor comprensión del mundo de sus hijos (Kumpfer et al., 2018; Kumpfer \& Magalhaes, 2018).

Finalmente, cabe mencionar que la presente investigación no está exenta de limitaciones, y una de ellas puede ser la muestra, que no es representativa de toda la variabilidad social nacional, pues no incluye los sectores rurales $\mathrm{u}$ otros contextos con mayor diversidad socioeconómica. Por 
126

otro lado, en este estudio tampoco se ha contemplado la inclusión de otros tipos de administración educacional del país - como son los colegios subvencionados particulares y particulares o privados-, lo cual podría otorgar mayor variabilidad a los resultados. Por último, es sabido que este tipo de estudio de corte transversal imposibilita la inferencia de causalidad, de manera que solo aporta evidencia de un único momento en el tiempo.

\section{Conclusiones}

Los resultados del presente estudio permiten validar la Escala de Fortalezas Familiares desarrollada por Olson et al. (1983), en su versión en español adaptada y validada por Sanz et al. (2002), en una población chilena.

En este sentido, los resultados permiten subsanar un vacío empírico existente en el contexto científico chileno, por lo que se consideran relevantes para el desarrollo de futuras investigaciones que posibiliten un mayor conocimiento del trabajo con familias. De hecho, esta versión chilena de la Escala de Fortalezas Familiares tiene especial relevancia para profesionales, equipos y programas de apoyo, pues permite ahondar en los procesos de intervención en áreas críticas de prevención y promoción en torno a fortalezas y recursos protectores orientados a la crianza respetuosa y afectiva, así como al mejoramiento de la calidad de vida familiar, especialmente con familias y grupos vulnerables.

Por otro lado, es importante mencionar que los resultados que se han obtenido a nivel internacional con la aplicación del enfoque de FF han facilitado una nueva forma de abordar no solo la comprensión de los complejos fenómenos que involucra la interacción entre los miembros de la familia mediante una propuesta que revaloriza las capacidades intrínsecas para sobrellevar los desafíos de la vida, sino que además han permitido avanzar en nuevas propuestas de intervención que incluyen aspectos que ponen de relieve las habilidades, cualidades y destrezas que sirven como soporte frente a la adversidad que muchas familias en contextos vulnerables vivencian.

Adicional a ello, a través del presente estudio se presenta un instrumento que puede complementar otras escalas validadas en Chile (Bello-Escamilla et al., 2017; Cumsille et al., 2014; Gubbins \& Ibarra, 2016; Rivadeneira \& López, 2017; Rivadeneira-Valenzuela et al., 2021) y que es especialmente relevante para la intervención con familias, puesto que considera la autoeficacia y la adaptación como elementos que pueden ayudar a hacer frente a la adversidad y a elementos estresores. Además, la aplicación de esta versión validada puede aportar evidencia en ámbitos como: (a) los procesos y mecanismos de afrontamiento que utilizan las familias para resolver las dificultades y desafíos cotidianos; (b) los recursos para desarrollar programas de intervención bajo el enfoque de fortalezas; y (c) los insumos de monitoreo del progreso de las familias que se encuentran adscritas a programas.

Como conclusión, los autores concuerdan en que un enfoque basado en fortalezas familiares puede ser eficaz, especialmente en poblaciones marginadas en las que los enfoques tradicionales de intervención se han centrado en las deficiencias del individuo y la familia. Dichos enfoques tradicionales suelen perpetuar la estigmatización y no permiten avanzar en el fortalecimiento de las potencialidades que tienen las familias para superar los desafíos asociados a la vulnerabilidad. Sin duda, se requiere de nuevos elementos que permitan, por tanto, que los equipos multidisciplinarios adopten nuevas concepciones más respetuosas, y que promuevan el desarrollo de las personas y las familias.

\section{Referencias}

Alavi, M., Visentin, D. C., Thapa, D. K., Hunt, G. E., Watson, R., \& Cleary, M. (2020). Chi-square for model fit in confirmatory factor analysis. Journal of Advanced Nursery, 76(9), 2209-2211. https://doi.org/10.1111/jan.14399

Bagur, S., \& Verger, S. (2020). Evidencias y retos de la atención temprana: El modelo centrado en la familia. Siglo Cero, 51(4), 69-92. https://doi.org/10.14201/scero20205146992

Baker, A., \& van Woerkom, M. (2018). Strengths use in organizations: A positive approach of occupational health. Canadian Psychology, 59(1), 38-46. https://doi. org/10.1037/cap0000120

Bandura, A. (2000). Exercise of human agency through collective efficacy. Current Directions in Psychological Science, 9(3), 75-78. https://doi.org/10.1111/1467-8721.00064

Bello-Escamilla, N., Rivadeneira, J., Soto-Caro, A., ConchaToro, M., \& Díaz, X. (2017). Escala de calidad de vida familiar: Validación y análisis en población chilena. Universitas Psychologica, 16(4), 1-10. https://doi.org/10.11144/ Javeriana.upsy16-4.ecvf

Churchill, S., \& Kieckhefer, G. M. (2018). One Year Followup of Outcomes from the Randomized Clinical Trial of the Building on Family Strengths Program. Maternal and Child Health Journal, 22(6), 913-921. https://doi.org/10.1007/ s10995-018-2467-4

Cumsille, P., Martínez, M. L., Rodríguez, V., \& Darling, N. (2014). Análisis psicométrico de la escala parental breve (EPB): Invarianza demográfica y longitudinal en adolescentes chilenos. Psykhe, 23(2), 1-14. https://doi.org/10.7764/ psykhe.23.2.665

De la Barra, M. F., Vicente, P. B., Saldivia, B. S., \& Melipillán, A. R. (2012). Estudio de epidemiología psiquiátrica en niños y adolescentes en Chile. Estado actual. Revista 
Médica Clínica Las Condes, 23(5), 521-529. https://doi. org/10.1016/S0716-8640(12)70346-2

Davenport, E., Kazman, J., \& Deuster, P. (2014). Family functioning and stress in African American families: A strength-based approach. Journal of Black Psychology, 41(2), 144-169. https://doi.org/10.1177\%2F0095798413520451

Drummond, J., Kysela, G.M., McDonald, L., \& Query, B. (2002). The family adaptation model: examination of dimensions and relations. The Canadian Journal of Nursing Research, 34(1), 29-46. https://cjnr.archive.mcgill.ca/ article/view/1748/1745

Figueroa, A. E. J., Subiabre, N. P. M., \& Fuentes, P. A. O. (2011). Satisfacción familiar, apoyo familiar y conflicto trabajo-familia en una muestra de trabajadores chilenos. Avances en Psicología Latinoamericana, 29(2), 317-329. https://revis tas.urosario.edu.co/index.php/apl/article/view/1165

Fitzgerald, H., Weatherson, D., \& Mann, T. (2011). Infant mental health: An interdisciplinary framework for early social and emotional development. Current Problems in Pediatric and Adolescent Health Care, 41, 178-182. https://doi. org/10.1016/j.cppeds.2011.02.001

Fruhauf, C., Yancura, L., Greenwood-Junkermeier, H., Riggs, N., \& Fox, A. (2020). The importance of family-focused and strengths-based approaches to interventions for grandfamilies. Journal of Family Theory \& Review, 12(4), 478-991. https://doi.org/10.1111/jftr.12395

Gardner, D. L., Huber, C. H., Steiner, R.,Vazquez, L. A., \& Savage, T. A. (2008). The development and validation of the inventory of family protective factors: A brief assessment for family counseling. The Family Journal, 16(2), 107-17. https://doi.org/10.1177/1066480708314259

Ghaffari, M. (2016). Spiritual well-being and dyadic adjustment: mediator effects for Family Strengths. Iran Journal of Psychiatry Behavior Science, 10(3), 1-8. https:// doi.org/10.17795/ijpbs-1699

Gilliam, C. M., \& Steffen, A. M. (2006). The relationship between caregiving self-efficacy and depressive symptoms in dementia family caregivers. Aging and Mental Health, 10(2), 79-86. https://doi.org/10.1080/13607860500310658

Greeff, A. P. (2000). Characteristics of families that function well. Journal of Family Issues, 21(8), 948-962. https://doi. org/10.1177\%2F019251300021008001

Gubbins, V., \& Ibarra, S. (2016). Estrategias educativas familiares en enseñanza básica: análisis psicométrico de una escala de prácticas parentales. Psykhe, 25(1), 1-17. https:// dx.doi.org/10.7764/psykhe.25.1.773

Hair, J. F., Black, W. C., Babin, B. J., \& Anderson, R. E. (2014). Multivariate Data Analysis: Pearson New International Edition (7. ${ }^{\mathrm{a}}$ ed.). Essex.

Julien-Chinn, F., Cotter, K., Hayes, M., Geiger, J., \& Lietz, C. (2017). Examining risk, strengths, and functioning of foster families: Implications for strengths-based practice. Journal of Family Social Work, 20(4), 306-321. https://dx.doi.org/1 $0.1080 / 10522158.2017 .1348111$
Kaiser, H. F., \& Rice, J. (1974). Little jiffy, Mark IV. Educational and Psychological Measurement, 34(1), 111-117. https:// doi.org/10.1177/001316447403400115

Kieckhefer, G., Trahms, C., Churchill, S., Kratz, L., Uding, N., \& Villareale, N. (2014). A Randomized clinical trial of the building on family strengths program: An education program for parents of children with chronic health conditions. Maternal and Child Health Journal, 18, 563-574. https:// doi.org/10.1007/s10995-013-1273-2

Kline, R. B. (2016). Principles and Practice of Structural Equation Modeling. Guilford Publications. (4. ${ }^{\mathrm{a}}$ ed.). New York.

Kumpfer, K., Scheir, L., \& Brown, J. (2018) Strategies to avoid replication failure with evidence- based prevention interventions: Case examples from the strengthening families program. Evaluation \& the Health Professions, 43(2), 1-34. https://doi.org/10.1177\%2F0163278718772886

Kumpfer, K., \& Magalhaes, C. (2018). Strengthening families program: An evidence-based family intervention for parents of high-risk children and adolescents. Journal of Child \& Adolescent Substance Abuse, 7(3), 174-179. https://doi.org/ 10.1080/1067828X.2018.1443048

Masten, A. S. (2018). Resilience theory and research on children and families: Past, present, and promise. Journal of Family Theory \& Review, 10(1), 12-31. https://doi.org/10.1111/ jftr. 12255

McKelvey, L., Schiffman, R., Brophy-Herb, H., London, E., Fitzgerald, H., Reischl, T., Hawver, S., \& Cunningham, M. (2015). Examining long-term effects of an infant mental health home-based early head start program on family strengths and resilience. Infant Mental Health Journal, 36(4), 353-365. https://doi.org/10.1002/imhj.21518

Montero, I., \& León, O. G. (2005). Sistema de clasificación del método en los informes de investigación en Psicología. International Journal of Clinical and Health Psychology, 5, 115-127. http://www.aepc.es/ijchp/articulos_pdf/ijchp136.pdf

Olson, D., \& Olson, A. (2000). Empowering couples. Building on your strenghts (2. ${ }^{\mathrm{a}}$ ed.). Life Innovations.

Olson, D.H., Larsen, A., McCubbin, H.I. (1982). Family Strengths. En D.H Olson, H.I McCubbin, H. Barnes, A. Larsen, M. Muxen, \& M. Wilson (Eds.). Family inventories: Inventories used in a national survey of families across the family life cycle (p. 78-92). St. Paul, MN: University of Minnesota.

Peña, Y. N., \& Molina, A. S. (2013). Caracterización de la salud familiar de una localidad para orientar un plan de cuidado. Revista Cubana de Enfermería, 29(2), 77-88. http://www. revenfermeria.sld.cu/index.php/enf/article/view/49/51

R Core Team (2018). $R$ : A language and environment for statistical computing. R Foundation for Statistical Computing. https://www.R-project.org/ 
Revelle, W. (2018). Psych: Procedures for personality and psychological research (Software version 1.8.4). Northwestern University.

Riquelme, E., Rojas, A., \& Jiménez, A. (2012). Equilibrio trabajo-familia, apoyo familiar, autoeficacia parental y funcionamiento familiar percibidos por funcionarios públicos de Chile. Trabajo y Sociedad, XVI(18), 203-215. https:// www.unse.edu.ar/trabajoysociedad/18\%20JIMENEZ\%20 ET\%20AL\%20Equilibrio\%20trabajo\%20familia.pdf

Rivadeneira-Valenzuela, J., Soto-Caro, A., Bello-Escamilla, N., Concha-Toro, M., \& Díaz, X. (2021). Estilos parentales, sobrepeso y obesidad infantil: Estudio transversal en población infantil chilena. Sociedad Chilena de Nutrición, 48(1), 1830. http://dx.doi.org/10.4067/S0717-75182021000100018

Rivadeneira, J., \& López, M. A. (2017). Escala de comunicación familiar: validación en población adulta chilena. Acta Colombiana de Psicología, 20(2), 116-126. http://www. dx.doi.org/10.14718/ACP.2017.20.2.6

Roque, M., \& Acle, G. (2012). Resiliencia materna, funcionamiento familiar y discapacidad intelectual de los hijos en un contexto marginado. Universitas Psychologica, 12(3), 811820. https://doi.org/10.11144/Javeriana.UPSY12-3.rmff

Rossel, Y. (2012). Lavaan: An R package for structural equation modeling and more. Version 0.5-12 (Beta). Journal of Statistical Software, 48(2), 1-36. https://users.ugent. be/ yrosseel/lavaan/lavaanIntroduction.pdf

Salas, M. I., Gattas, V., Ceballos, X., \& Burrows, R. (2010). Tratamiento integral de la obesidad infantil: efecto de una intervención psicológica. Revista Médica de Chile, 138(10), 1217-1225. http://dx.doi.org/10.4067/ S0034-98872010001100002

Samson, F. L. (2018). An association between multiculturalism and psychological distress. PLOS One, 13(12), e0208490. https://doi.org/10.1371/journal.pone.0208490

Sánchez, R., Letiecq, B., \& Ginsberg, M. (2019). An Integrated Model of Family Strengths and Resilience: Theorizing at the Intersection of Indigenous and Western Paradigms. Journal of Family and Theory Review, 11(4), 561-575. https://doi. org/10.1111/jftr.12351

Sanders, M., \& Woolley, M. (2005). The relationship between maternal self-efficacy and parenting practices: Implications for parent training. Child: Care, Health and Development, 31(1), 65-73. https://doi. org/10.1111/j.1365-2214.2005.00487.x

Sanz, M., Iraurgi, I., \& Martínez-Pampliega, A. (2002). Evaluación del funcionamiento familiar en toxicomanías: adaptación española y características de adecuación métrica del FAP/FACES-IV. En I. Iraurgi, \& F. González-Said (Eds.), Instrumentos de Evaluación en Drogodependencias (pp. 403-434). Aula Médica.

Sanz, M. (2003). El funcionamiento familiar de los drogodependientes a lo largo de un tratamiento. (Tesis doctoral no publicada). Universidad de Deusto.
Schumm, W. R, Bollman, S. R, Jurich, A. P, \& Hatch, R. C. (2001). Family strengths and the Kansas Marital Satisfaction Scale: A factor analytic study. Psychological Reports, 88(3), 965-973. https://doi.org/10.2466/pr0.2001.88.3c.965

Sheridan, S., Moen, A., \& Bhatia, S. (2017). Family-Centered Positive Psychology: A Framework for Research-based Practices. En C. R. Snyder, S. J. López, L. M. Edwards \& S. C. Marqués (Eds), The Oxford Handbook of Positive Psychology (3. ${ }^{\text {a }}$ ed., pp. 1-24). University of Kansas.

Soler, M. I., Meseguer, M., \& García, M. (2016). Propiedades psicométricas de la versión española de la escala de resiliencia de 10 ítems de Connor-Davidson (CD-RISC 10) en una muestra multiocupacional. Revista Latinoamericana de Psicología, 48(3), 159-166. https://doi.org/10.1016/j. rlp.2015.09.002

Spielman, V., \& Taubman-Ben-Ari, O. (2009). Parental selfefficacy and stress-related growth in the transition to parenthood: a comparison between parents of pre- and fullterm babies. Health Soc Work, 34(3), 201-212. https://doi. org/10.1093/hsw/34.3.201

Souza, J., \& Aparecida, M. (2019). Problemas emocionales y comportamentales en los niños: asociación entre el funcionamiento familiar, la coparentalidad y la relación conyugal. Acta Colombiana de Psicología, 22(1),82-94. https://doi. org/10.14718/acp.2019.22.1.5

Thompson, P. A., \& Schrodt, P. (2015). Perceptions of joint family storytelling as mediators of family communication patterns and family strengths. Communication Quarterly, 63(4), 405-426. https://doi.org/10.1080/01463373.2015.10 58286

Valdez, C., Abegglen, J., \& Hauser, C. (2013). Fortalezas Familiares Program: Building sociocultural and family strengths in Latina women with depression and their families. Family Process, 5(3), 378-393. https://doi.org/10.1111/ famp. 12008

Valdivia, M., Silva, D., Sanhueza, F., Cova, F., \& Melipillán, R. (2015). Prevalencia de intento de suicidio adolescente y factores de riesgo asociados en una comuna rural de la provincia de Concepción. Revista Médica de Chile, 143(3), 320328. http://dx.doi.org/10.4067/S0034-98872015000300006

Vega, M.T., \& de Dios, M. Y. (2006). Beneficios psicosociales de los grupos de apoyo: su influencia en los estilos de socialización familiar. Intervención Psicosocial, 15(2), 233-244. https://journals.copmadrid.org/pi/archivos/102111.pdf

Vicente, B., Saldivia, S., De la Barra, F., Melipillán, R., Valdivia, M., \& Kohn, R. (2012). Salud mental infanto-juvenil en Chile y brechas de atención sanitarias. Revista Médica de Chile, 140(4), 447-457. http://dx.doi.org/10.4067/ S0034-98872012000400005

Walsh, F. (2016). Family resilience: A developmental systems framework. European Journal of Developmental Psychology, 13(3), 313-324. https://doi.org/10.1080/17405 629.2016 .1154035 
Wolf, E., Harrington, K., Clark, S., \& Miller, M. (2013). Sample size requirements for structural equation models: an evaluation of power, bias, and solution propriety. Educational and Psychological Measurement, $X X(\mathrm{X}), 1-22$. https://doi. org $/ 10.1177 \% 2 \mathrm{~F} 0013164413495237$

Yeung, J., \& Chan, Y. C. (2011). The mediating role of parental psychological health in Chinese families in an impoverished context in Hong Kong. Journal for General Social Issues, 20(4), 1085-1100. https://doi.org/10.5559/di.20.4.08
Yeung, J., Lee, S., Lee, E., \& DeFrain, J. (2012). Development and validation of an chinese family strengths measure for family services intervention in Hong Kong. Revista de Cercetare si Interviere Sociala, 36, 7-30. https://www.rcis. ro/images/documente/rcis36_01.pdf 